\title{
An Adaption of OSA-CBM Architecture for Human-Computer Interaction through Mixed Interface
}

\author{
Danúbia Espíndola $^{*+}$, Luca Fumagalli ${ }^{\S}$, Marco Garetti ${ }^{\S}$, Silvia Botelho ${ }^{+}$and Carlos Pereira* \\ ${ }^{*+}$ Department of Electrical Engineering, \\ Federal University of Rio Grande do Sul, Porto Alegre, Brazil \\ \{danubia, cpereira\}@ece.ufrgs.br \\ ${ }^{+}$Centro de Ciências Computacionais, \\ Federal University of Rio Grande, Rio Grande, Brazil \\ \{danubiaespindola,silviacb\}@furg.br \\ ${ }^{\S}$ Department of Management, Economics and Industrial Engineering, \\ Politecnico di Milano ${ }^{\S}$, Milano, Italy \\ \{lucal.fumagalli,marco.garetti\}@polimi.it
}

\begin{abstract}
The technological advances in graphics processing have provided an emerging transformation in the methods of interaction and visualization computational systems. The possibility to access, to visualize and to understand the information in the right place at the right time has been the main focus of mixed reality research in industry applications. These technologies have provided a potential solution in industrial environment especially regarding the downtime reduction of critical equipment in condition based maintenance CBM systems. Currently, there are many studies and projects to increase their use in maintenance processes. However, there are few researches regarding the interaction of the "HumanComputer Interface" module with other modules of the maintenance OSA-CBM architecture (Open System Architecture for Condition Based Maintenance). Another significant aspect is that most studies do not address the issue of data management (different formats) in mixed environments. Thus, this paper proposes a methodology for management and visualization of information using mixed reality for interaction with all modules of the OSA-CBM architecture.
\end{abstract}

\section{INTRODUCTION AND BACKGROUND}

The Mixed Reality (MR) is an area of Virtual Reality (VR) that involves real and virtual components in the same scene presented to the user to improve the human-computer interaction. There are two types of MR, the Augmented Reality (AR) which has a predominance of real environment and the Augmented Virtuality (AV) where the virtual environment is predominant. Through the use of VR devices such as transparent glasses, tablets or PDAs, it is possible to view the real environment, being guided by virtual information overlaid in an interface in real-time [1].

The dissemination about the using of MR in visualization technologies are a reality on mobile phones, gaming industry, smart homes projects, entertainment movies, medicine applications, learning environments and so on. However, the mixed visualization is not yet a common practice in industry.
In most cases, only large automotive and aerospace industries invest on the implementation of these technologies [2][3]. There were several reasons for this, mainly due to the cost of the equipment and related software. However the situation is changing rapidly due to the improvements of ICT, together with the parallel decrease of its cost. Today, there is the lack of standards, architectures and models in the context of Human-machine interfaces to manage information in industry applications. Solutions in this sense decrease the time to deployment of these applications that is one of the main reasons preventing their use to all industry.

Two huge European projects in the mixed reality applied to industry are the most cited: the project ARVIKA (Augmented Reality für Entwicklung, Produktion und Service) [4] and the project AMIRE (Authoring Mixed Reality) [5]. ARVIKA's contribution is mainly the presentation and discussion of several AR potentialities in the fields of development (design), production (assembly and assembly/disassembly optimization) and services (inspection and diagnosis). Assembly, inspection and diagnostic processes are the fields of interest for the maintenance activity, but no specific details about the implementation of these applications were found. AMIRE proposes an object-oriented framework for the use of MR by users who are not programming experts. The project provides a repository of components for the virtual content generation, however to integrate data from other systems is necessary to adapt the available codes. After these projects a lot of research started to discuss the advantages and disadvantages of using MR/AR in industry (more details can be found in [6] [7] [8] [9]).

The focus of this work is the use of mixed reality for maintenance activities on critical equipment in industry. Our main goal is to reduce downtime of equipment during maintenance activity. To carry out this task efficiently, it is 
necessary the effective use of information, i.e., to extract and to bring the right information at the right time. However, information management is not a trivial task, since it involves different systems and data formats. On the other hand, the use of CAD tools including support to virtual modeling has become widespread in the industry. This implies that most of the work is ready to create interaction mixed environment.

Thus, in order to develop the present research, standards and models in information management used for maintenance activities were investigagated. The condition-based maintenance approach (CBM) applies predictive techniques to avoid unplanned breakdown of equipment. These systems predict where, when and why the failure will occurs [10]. CBM systems are often used in equipment which breakdown impacts directly the production line. The approach here proposed will help not only in the visualization technique, but also in the interaction with several modules of OSA-CBM architecture.

The goals of this research are:

1. To describe an approach for the visualization and the interaction in CBM systems.

2. To design a methodology to implement the proposed approach.

3. To validate the proposal in a case study in progress.

4. To discuss and to plan future work.

\section{THE APPROACH}

Analyzing the researches on the formalization and standardization for condition based maintenance technologies, the MIMOSA and OSA-CBM organizations are highlighted [11]. The MIMOSA (Machine Information Management Open System Alliance) is a nonprofit organization that provides specifications for enterprise application integration (OSA-EAI- Open System Architecture for Enterprise Application Integration) and condition based maintenance (CBM) [12]. The OSA-CBM specification divides in seven modules the CBM systems and its architecture, see the description in Lebold et al. [13].

The idea of this work is to interact through mixed interface with the seven modules of the OSA-CBM architecture in order to bring the necessary information and support maintenance activities, reducing the time to intervene. So, a methodology is proposed for the deployment of mixed reality in CBM systems. The figure 1 shows the Human-Computer module interact with all the others and not only with the Automatic Decision Reasoning, Complex System and Data Acquisition modules.

The proposed idea, according to figure 1, focuses on expanding the interaction of the Human-Computer Interface module. Basically, the architecture describes the health monitoring of the equipment through the acquisition and manipulation of data. Data are acquired through sensors and the decision making is performed based on diagnostic results (health assessment) and prognostic activity provided by signal processing techniques. Each one of these functions is separated in modules as described in figure 1.

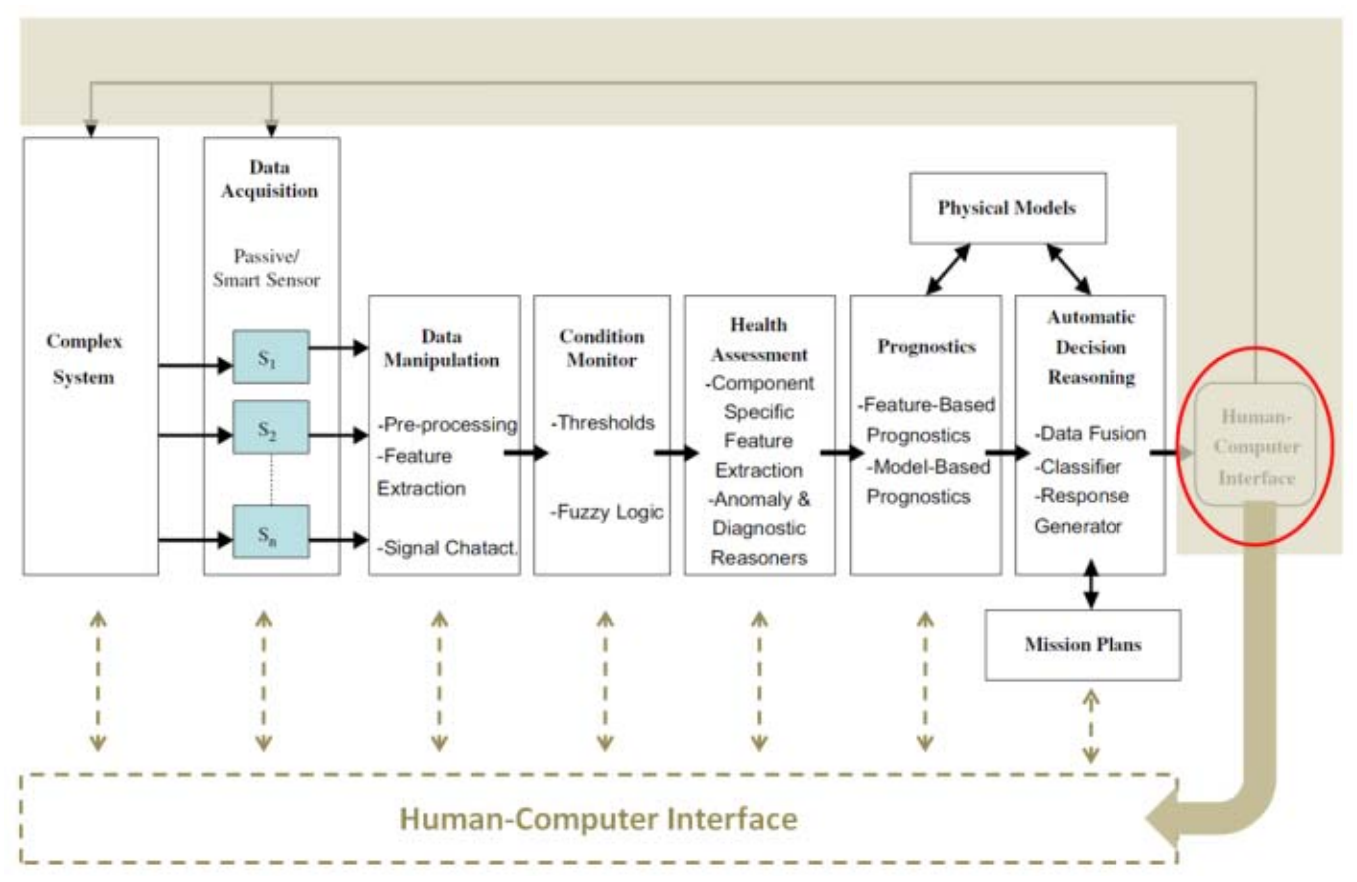

Fig. 1. The idea (The module's extension HC Interface in the OSA-CBM architecture [13]). 
The interaction proposal of Human-Computer Interface module implemented through the MR techniques is represented by seven bi-directional arrows in figure 1 . The directions and the information content are described in table 1 below. The $\mathrm{H} \rightarrow \mathrm{C}$ symbol represent the interaction from operator to computer, and the $\mathrm{C} \rightarrow \mathrm{H}$ is the opposite, represents the interaction from computer to operator.

TABLE I

INTERACTION DESCRIPTION IN THE OSA-CBM ARCHITECTURE

\begin{tabular}{|c|c|}
\hline SETA & INTERACTION \\
\hline I. & $\begin{array}{l}(\mathrm{H} \rightarrow \mathrm{C}) \text { - operation of the machine (maintenance tasks) } \\
(\mathrm{C} \rightarrow \mathrm{H}) \text { - mixed interface (mixed visualization) }\end{array}$ \\
\hline II. & $\begin{array}{l}(\mathrm{H} \rightarrow \mathrm{C}) \text { - to configure sensors } \\
(\mathrm{C} \rightarrow \mathrm{H}) \text { - txt and graphical files from data acquisition }\end{array}$ \\
\hline III. & $\begin{array}{l}(\mathrm{H} \rightarrow \mathrm{C}) \text { - to configure and to chose the feature } \\
\text { extraction methods } \\
(\mathrm{C} \rightarrow \mathrm{H}) \text { - 2D graphical about signal feature }\end{array}$ \\
\hline IV. & $\begin{array}{l}(\mathrm{H} \rightarrow \mathrm{C}) \text { - to configure the monitoring thresholds } \\
(\mathrm{C} \rightarrow \mathrm{H}) \text { - 2D graphical and virtual texts about } \\
\text { monitoring }\end{array}$ \\
\hline $\mathrm{V}$ & $\begin{array}{l}(\mathrm{H} \rightarrow \mathrm{C}) \text { - requisition of component diagnostic and } \\
\text { parameters configuration } \\
(\mathrm{C} \rightarrow \mathrm{H}) \text { - 2D graphical and virtual information about } \\
\text { the component health }\end{array}$ \\
\hline VI. & $\begin{array}{l}(\mathrm{H} \rightarrow \mathrm{C}) \text { - requisition of component prognostic and } \\
\text { parameters configuration for model based and feature } \\
\text { based prognostic } \\
(\mathrm{C} \rightarrow \mathrm{H}) \text { - 2D graphical and virtual information about } \\
\text { the component prognostic }\end{array}$ \\
\hline VII. & $\begin{array}{l}(\mathrm{H} \rightarrow \mathrm{C}) \text { - requisition of instruction guide; update in } \\
\text { data maintenance historical } \\
(\mathrm{C} \rightarrow \mathrm{H}) \text { - texts, graphical and videos about maintenance } \\
\text { procedure }\end{array}$ \\
\hline
\end{tabular}

After the idea definition and description of the types of interaction in the modules of the architecture, it is now more understandable the need of the integration between maintenance support and virtual systems. However, the following challenges about the components identification arises:

- how to navigate the virtual components of the equipment?

- $\quad$ how to relate the virtual and maintenance information to each real component?

These issues led us to identify the need of a model for machine hierarchical description. In this sense, it was identified that the use of CAD models can provide XML description models of the equipment can assist in the information relationship definition. This way the figure 2, where the intersection domain among three systems (CAD, virtual and maintenance systems) is represented, shows our approach to implement the idea.

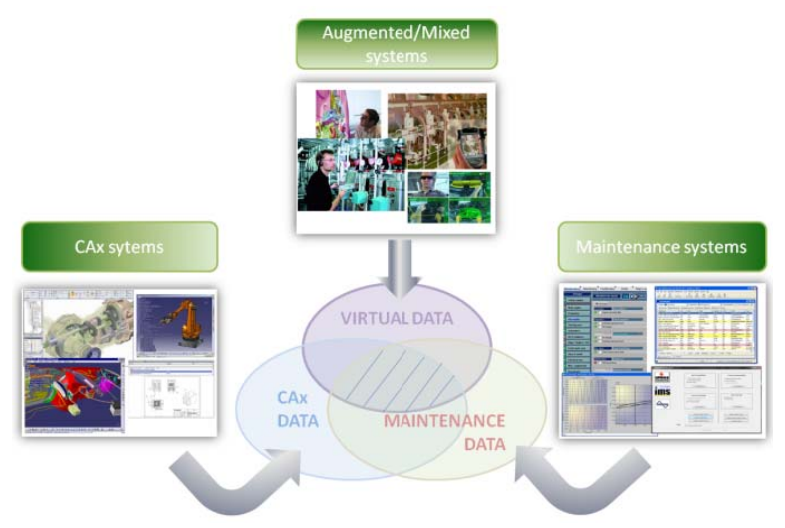

Fig. 2. The approach (CAD, CBM and $\mathrm{AR}$ data integration )

The approach aims at to integrating data from maintenance, $\mathrm{CAD}$ and virtual systems to implement a mixed interface of interaction in OSA-CBM architecture. To implement this approach is proposed a development methodology described in next section 3 .

\section{THE METHODOLOGY}

The methodology describes the stages of implementation for the mixed visualization proposed on the OSA-CBM architecture. The implementation process is divided into six stages acording to figure 3 .

The stages of methodology are:

\section{Interface configuration \\ 2. Environment capture \\ 3. Tracking \\ 4. Relationship description \\ 5. Information extraction \\ 6. Content generation}

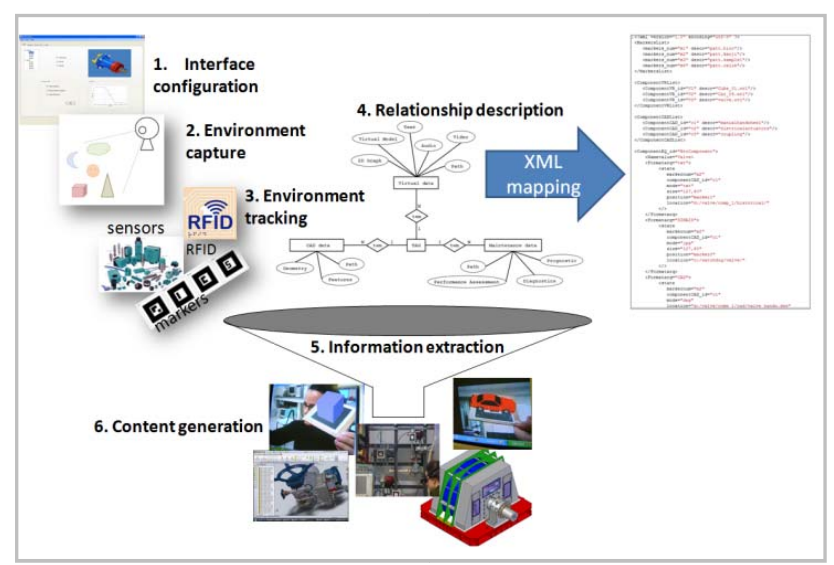

Fig. 3. The methodology. 
The first stage for the implementation of the mixed visualization is the interface configuration. In this stage, the operator will define, guided by virtual instructions, the path of virtual, CAD, and maintenance data for each marker. After the definition of the relationship between marker and paths, the capture system is activated.

Then the capture phase starts. For the image acquisition from real environment is necessary to use a hardware and software device tailored for such function. Video cameras or webcams and software such as Vision SDK and DirectShow can be used for this purpose.

Once the capture phase is started, the tracking process of the real environment begins, in order to overlay virtual components. The position tracking for the placement of the virtual objects can use different techniques such as: image processing with the use of markers or computing vision without the use of markers (markerless or feature based tracking technique). However the high complexity of markeless techniques makes difficult their use. In addition to these techniques, the use of RFID and sensors has started to be deployed in ubiquitous and mixed reality environments.

Both technologies have advantages and disadvantages. Markless techniques have high complexity for implementation (due to mathematical calculations based on CAD models), it increase the time to deployment. On the other hand, the use of markers has the problem of occlusion and placement of these markers in the environment. Perhaps the use of RFID for equipment identification in the plant associated with markeless techniques for identify components of machine would be the next horizon for future research in maintenance visualization.

Once the interface has been configured and the environment captured and tracked, the next stage is the relationship description. At this phase, the interface generates automatically an XML description of the relationship between different data types. This file is described based on user configuration and on XML generated from the CAD model.

The information extraction stage is directly related to the previous step. This stage is based on user requests and in the XML description model. The XML file is used as a searching map for content generation.

Finally, the content generation phase is responsible for presenting the information in the mixed interface. This information can be presented in two forms: autonomous or guided. The automatic generation of content is triggered in failure cases identified by the CBM system. The guided visualization is performed according to user request. The request can be run through voice commands or mobile devices [14] and also by conventional devices such as mouse or keyboard [15].

In the next section, the proposed methodology is validated through the implementation in a case study in offshore industry.

\section{IMPLEMENTATION}

The figure 4 represents the software infrastructure used to implement the proposal.

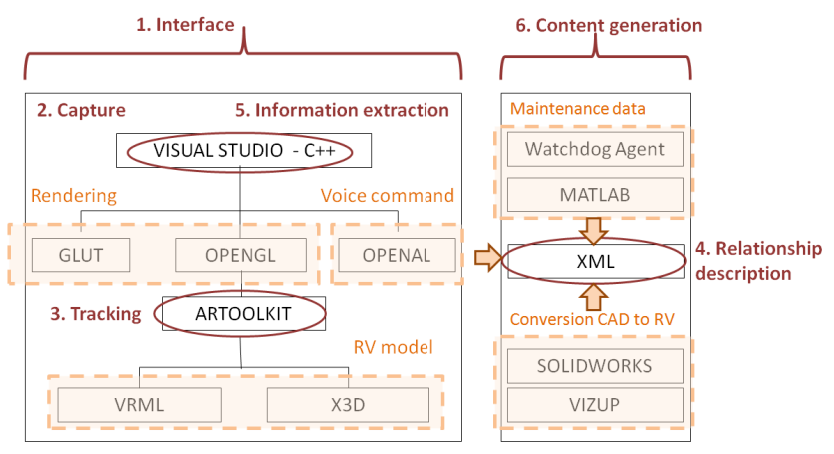

Fig. 4. The software infrastructure.

The interface has been implemented with the $\mathrm{C}++$ language on Visual Studio platform. The capture and information extraction stages also have been implemented in $\mathrm{C}++$. The ARToolKit library[16] is used to optical tracking through computer vision techniques allowing identify and estimate in real time the position and orientation of a marker. These markers are symbols printed on paper and placed on the real equipment to positioning.

For the 3D modeling the use of VRML [17] and X3D [18] was chosen, once the current CAD software have CAD-RV conversion resources. This will facilitate the task and reduce the time of the $3 \mathrm{D}$ virtual modeling. SOLIDWORK was used to conversion CAD-RV and the VIZUP to reduce the number of polygons in complex models before to convert in wrl format. The GLUT and OPENGL [19] libraries are used to rendering the scene.

The CBM system used to validate the proposal was the Watchdog Agent [20]. This system consists of prognostic algorithms embedded with a group of software tools to predict components and systems failure. This degradation calculation is based on sensor readings which measure the critical properties of the process/machinery. The system supplies a MATLAB tool and the codes can be accessed and manipulated. The outputs supplied by the tool are 2D graph of data manipulation, condition monitoring, health assessment-diagnostics and prognostics.

Finally, the relationship among $\mathrm{CAD}$, virtual and maintenance data are described in XML format. The XML language generates a nodes hierarchical model regarding the equipment components. Each node in the XML model contains the virtual, maintenance and $\mathrm{CAD}$ information supplied by several systems. With that, the interface can map the information of the several systems of intuitive and explanatory way. 


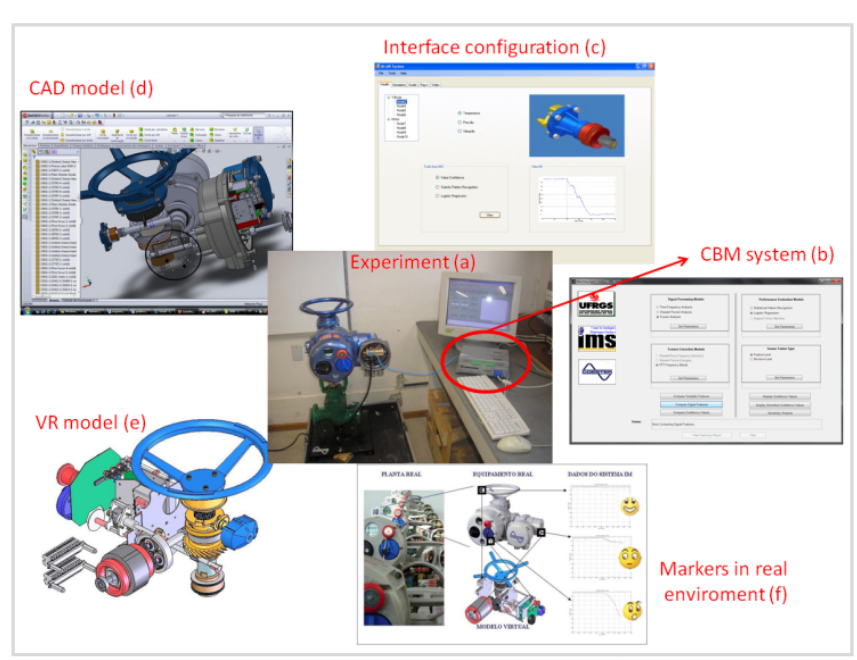

Fig. 5. The case study.

The case study was carried out in CRS Actuator from Coester industry. The figure 5 (a) presents the physical implantation of the CBM system using the Watchdog Agent. In this experiment was introduced a rubber pipe in the valve entrance to simulate the fault in the opening and closing valve. The Matlab toolbox (b) supplied by Watchdog system provide CBM techniques. With this system some graphs were generated for results analysis and integrated in the configuration interface (c). The virtual model of actuator (e) was created from CAD model (d) using SolidWorks tool. Finally the figure 5 (f) represents the simulation of the use of markers to mixed interface deployment in industrial environment. The next step will be test the using of HMD (Head Mounted Displays) by maintenance operators on the factory floor.

\section{CONCLUSIONS AND FUtURE WORK}

This study identified the possibility of using mixed reality as visualization technology to support the condition based maintenance systems. After this proposed idea, a plan of approach was outlined. The approach proposes a set of interactions through the domains intersection of three distinct systems. Finally an approach for implementing the methodology was proposed and validated in a case study of industrial actuators.

Although the experiment has not implemented the use of HMD immersive device on the factory floor, it was possible with monitors to visualize the mixed environment. Information from $\mathrm{CAD}$, virtual and maintenance models supported the interaction with the modules of the OSA-CBM architecture.

However, to make "plug and view" paradigm true, an appropriate formalization of concepts, models and architectures is necessary, in order to propose standards for the installation of mixed visualization systems in maintenance applications. Adaptable architectures should be proposed for use in devices such as: PDA, mobile phone and Tablets.
Challenges in the area of data repositories and communication should also be depth.

In this sense, future work of this study intend to focus on the development of theoretical and technical models, especially regarding integration and management data, as well as architecture to mixed visualization platform applied to industrial maintenance systems. Moreover tests with other information source from patterns and maintenance systems describe in PROMISE (PROduct lifecycle Management and Information tracking using Smart Embedded systems) [21] project and investigated in COMADEM (Condition Monitoring and Diagnostics Engineering Management) area will be analyzed.

\section{REFERENCES}

[1] R. Azuma, "A survey of augmented reality", Teleoper Virtual Environ 6(4):355-385, 1997.

[2] R. Schoenfelder and D. Schmalstieg, "Augmented Reality for Industrial Building Acceptance", In Proceedings of IEEE VIRTUAL REALITY, 2008.

[3] N. Zenati, et al., "Assistance to Maintenance in Industry Process Using an Augmented Reality System", In Proceedings of IEEE International Conference on Industrial Technology - KIT2004, 2004.

[4] W. Friedrich, D. Jahn and L. Schmidt, "ARVIKA - Augmented Reality for Development, Production and Service", In Proceedings of the International Symposium on Mixed and Augmented Reality (ISMAR'02) pp.3-4, 2002.

[5] P. Grimm, M. Haller, V. Paelke, S. Reinhold, C. Reimann and J. Zauner, "AMIRE - Authoring Mixed Reality" In The First IEEE International Augmented Reality Toolkit Workshop, Darmstadt, Germany, September, 2002.

[6] T. Schlegel, A. Srinivasan, M. Foursa, M. Bogen, R. Narayanan, D’Angelo D., D. Haidegger, I. Mezgar, J. Canou, D. Salle, F. Meo, J. Agirre and A. Praturlon, "INT-MANUS: Interactive Production Control in a Distributed Environment", In Proceedings of the 12th International Conference on Human-Computer Interaction (HCII), pp. 1150-1159, Bejing, China, July 2007.

[7] A. Makri, D. Arsenijevic, J. Weidenhausen, P. Eschler, D. Stricker, O. Machui, C. Fernandes, S. Maria, G. Voss and N. Ioannidis. "ULTRA: An augmented reality system for handheld platforms, targeting industrial maintenance applications", In Proceedings 11th International Conference On Virtual Systems And Multimedia, Ghent, Belgium, 2005.

[8] M. Foursa and et al., "INT-MANUS: revolutionary controlling of production processes", In Proceedings of ACM SIGGRAPH, New York, n.161, 2006.

[9] M. Fiorentino, G. Monno and A. E. Uva, "Tangible digital master for product lifecycle management in augmented reality", In Proceedings of the International Journal On Interactive Design And Manufacturing, Volume 3, Number 2, 121-129, 2009.

[10] L. Fumagalli, E. Jantunen, M. Garetti, and M. Macchi, "Diagnosis for Improved Maintenance Services: Analysis of Standards Related to Condition Based Maintenance", In Proceedings of the 4th World Congress on Engineering Asset Management pp. 288-297, Athens, Greece, 2009.

[11] A. Muller, A. Marquez and B. Iung, "On the concept of e-maintenance: Review and current research", In Reliability Engineering and System Safety, p. 1165-1187, 2007

[12] J. Mitchell, T. Bond, K. Bever and N. Manning, "MIMOSA-Four years later", Sound Vib pp. 12-21, 1998.

[13] M. Lebold and M. Thurston, "Open standards for condition-based maintenance and prognostic systems", In Proceedings of MARCON Fifth annual maintenance and reliability conference, Gatlinburg, USA, 2001.

[14] L. Fumagalli, E. Juntunen and M. Macchi, "Ontology of Mobile Maintenance Processes”, In MFPT, Dayton, USA, 2009. 
[15] D. Espíndola and C. Pereira, "A solution for integration of IM and MR data", In the Proceedings 11th International Conference on The Modern Information Technology in the Innovation Processes of the Industrial Enterprises, p. 423 - 430, Bergamo, 2009.

[16] H. Kato, M. Billinghurst and I. Poupyrev, "ARToolKit version 2.33 Manual", 2000.

[17] L. Ames, R. Nadeau and D. Moreland, "VRML Sourcebook - Second Edition”, In John Wisley and Sons USA, 1997.

[18] X3D. "Web3D Consortium",

2006. Disponível $\mathrm{em}:<\mathrm{http}: / /$ www.web3d.org/about/overview/>. Acesso em: $13 \mathrm{dez}$ 2010 .
[19] OPENGL, "Open Graphics Library - Silicon Graphics Interface”, 1997. Disponível em: < http://www.opengl.org/about/overview/>. Acesso em: 13 dez 2010.

[20] D. Djurdjanovic, J. Lee and J. Ni, "Watchdog Agent, an infotronicsbased prognostics approach for product performance degradation assessment and prediction", In Proceedings of Advanced Engineering Informatics - Elsevier, p.109-125, 2003.

[21] D. Kiritsis, "Ubiquitous product lifecycle management using product embedded information devices", In International Conference On Intelligent Maintenance Systems - IMS2004, 2004, Arles, France, 2004. 